\title{
Corela
}

Cognition, représentation, langage

HS-4 | 2006

Le parcours

\section{Parcours et interlexis (la relation de concession)}

\section{Catherine Filippi-Deswelle}

\section{OpenEdition}

\section{Journals}

Édition électronique

URL : http://journals.openedition.org/corela/1328

DOI : $10.4000 /$ corela.1328

ISSN : 1638-573X

\section{Éditeur}

Cercle linguistique du Centre et de l'Ouest - CerLICO

\section{Référence électronique}

Catherine Filippi-Deswelle, «Parcours et interlexis (la relation de concession) », Corela [En ligne], HS-4 | 2006, mis en ligne le 08 juin 2006, consulté le 02 mai 2019. URL : http:// journals.openedition.org/corela/1328; DOI : 10.4000/corela.1328

Ce document a été généré automatiquement le 2 mai 2019.

\section{(c) (i) (9)(2)}

Corela - cognition, représentation, langage est mis à disposition selon les termes de la licence Creative Commons Attribution - Pas d'Utilisation Commerciale - Partage dans les Mêmes Conditions 4.0 International. 


\title{
Parcours et interlexis (la relation de concession)
}

\author{
Catherine Filippi-Deswelle
}

\section{Définitions}

1 Avec les énoncés concessifs, la question de l'opération de parcours ne semble pas immédiatement pertinente. Commençons par rappeler quelques définitions bien connues dans la TOE élaborée par Antoine Culioli (cadre théorique dans lequel se situe la présente intervention).

Antoine Culioli définit l'opération de parcours en ces termes :

«L'opération de «parcours " consiste à parcourir toutes les valeurs assignables à

l'intérieur d'un domaine sans pouvoir s'arrêter à une valeur distinguée (ainsi :

« tout chien a quatre pattes »; « any dog barks »). ( (Culioli 1999, T. $3: 48$ )

«Nous avons appelé parcours cette opération qui consiste à parcourir toutes les

valeurs ou opérations possibles dans une des places d'une relation à $\mathrm{n}$ places, sans

(vouloir/pouvoir) distinguer telle ou telle d'entre elles. » (Culioli 1999, T. 3 :119)

Dans le cadre de sa «théorie des relations ", le linguiste évoque la question de l'issue au parcours :

« relation entre termes non stabilisés : on a alors une opération de parcours, c'est-

à-dire un trajet d'occurrence à occurrence, sans que l'on puisse s'arrêter à une valeur stable et assurée. Dans ce cas, on aura nécessairement recherche d'un site (processus de stabilisation): nous appellerons issue une telle position de stabilisation. On constitue ainsi une relation entre deux représentations, à savoir, un parcours et une issue. » (Culioli 1990, T.1:170)

Dans les énoncés concessifs, il ne s'agit pas de passer en revue les occurrences de la classe d'une notion quantifiabilisée sur lesquelles l'énonciateur ne peut ni ne veut s'arrêter, à savoir d'un parcours sans issue ; l'opération de parcours porte en effet sur des notions complexes (des lexis prédiquées ou encore des relations prédicatives) mises en contact, et est indissociable de l'aboutissement vers une issue concernant chacune d'elles: on travaille sur des propositions assertées, dont la référence est stabilisée. 
3 Ainsi, l'opération de parcours dont il est ici question se rattache au fonctionnement la modalité 1 de l'assertion (selon la typologie des modalités établie par A. Culioli) et a trait au balayage des deux valeurs I (pour l'Intérieur) et $\mathrm{E}$ (pour l'Extérieur) du domaine notionnel ${ }^{1}$ propre à chaque lexis prédiquée, $\mathrm{A}$ (la « subordonnée ») et $\mathrm{B}$ (la « principale »), mises en relation par le marqueur concessif selon l'ordre marqueur concessif $A, B$.

\section{Rappel sur l'opération d'assertion}

On est à la recherche d'une lexis prédiquée stabilisée sur les plans référentiel (paramètre $\mathrm{T}$ ) et intersubjectif (paramètre $\mathrm{S}$ ) de sorte qu'elle ne puisse faire l'objet d'aucune remise en cause énonciative, ni existentielle ni subjective. On part donc d'une position horsdomaine et il convient de combler le hiatus (la distance) entre le plan pré-modal et celui de la validité. On a "l'ordre oui, non qui structure le domaine notionnel, lorsqu'on parcourt les chemins de la came. Hors Intérieur/Extérieur $\rightarrow$ Intérieur $\rightarrow$ Extérieur $\rightarrow$ Retour à Hors Intérieur/Extérieur» (Culioli 1999, T. 3: 96; notre surlignage de «parcourt »).

Dans le cas de l'assertion tranchée entre I et $\mathrm{E}$, cela conduit à annuler toute distance entre les deux plans puisque l'on construit une double boucle d'identification sur une valeur et une seule, et donc une "référence stabilisée, grâce à l'élimination de toute altérité » (Culioli 1999, T. $3:$ 133).

Or l'opération d'assertion ne va pas de soi dans la configuration concessive car l'énonciateur comme le co-énonciateur ${ }^{2}$ sont appelés à se positionner face à la prise en compte de l'altérité : la construction de l'accès à tel chemin (vers I ou vers E), à savoir l'accessibilité de la valeur positive ou de la valeur négative de chaque lexis prédiquée, est centrale et se pose aussi bien sur le plan quantitatif (existence ou non de tel état de choses) que sur le plan qualitatif (conformité à telle représentation notionnelle typique sur le plan subjectif; valuation téléonomique dans la recherche de la « bonne » valeur par et pour le sujet, en lien avec la modalité 3 appréciative). Ainsi, chaque lexis prédiquée fait l'objet d'un travail d'évaluation énonciative sur les plans assertif et téléonomique par l'énonciateur d'une part et par le co-énonciateur d'autre part.

7 Dans l'antéposition de la lexis introduite par le subordonnant concessif, se construisent d'emblée, du fait du sémantisme adversatif de ce dernier, à la fois un conflit intersubjectif sur les états de choses respectivement prédiqués et la résolution de ce conflit au profit des choix retenus par l'énonciateur, à l'encontre de ceux attribués au co-énonciateur. L'énoncé concessif comporte donc les traces de ce nécessaire ajustement entre les sujets au niveau de leurs représentations physico-culturelles et de leurs normes personnelles. ${ }^{3}$ Nous allons à présent en étudier les marqueurs à partir de quelques énoncés en français et en anglais :

(1) «Bien que nous soyons en mai, il fait froid.

Nous avons beau être en mai, il fait froid. » (A. Culioli, $2002: 171$ )

(2) Although it was April, a freezing wind blew through the streets of the city.

(R. Dahl, cité par Deléchelle (1982) et repris par Delmas (1987 


\section{Relation concessive-adversative : quelle place pour l'opération de parcours dans la construction de l'altérité ?} beau (il a beau) sont l'image d'une assertion positive infiniment itérée » (Culioli 1999, T.2 : 50) et précise ailleurs le lien entre bien et " parcours »:

«bien, terme qualitativement positif, (de même que beau, d'ailleurs) est le marqueur d'une opération plus complexe qu'il n'y paraît. D'un côté, on construit un domaine, et l'on retrouve là, sur un mode symbolique, une relation connue entre / bien/ et existence, avoir, réalité délimitée. En second lieu, on parcourt la classe d'occurrences, ce qui se ramène à une itération ; puis l'on établit une relation : dans ces deux derniers cas, on peut à nouveau montrer le lien qui existe entre le caractère positif de bien et l'itération ou la mise en relation assertive." Avec «bien » : il y a "parcours sur un ouvert ; franchissement de frontière visé ». (Culioli 1990 , T. $1: 143 ; 152)$

Il semble qu'au départ, à un stade non nécessairement verbalisé, on ait affaire à un état de fait construit comme validé dans la situation: nous sommes en mai (A). Or dans la configuration concessive, on réintroduit la possibilité d'une instabilité quantitative et qualitative dans le domaine de validation, dans la mesure où des énonciateurs distincts sont amenés respectivement à se prononcer sur le bien fondé de telle ou telle validation.

Le domaine parcouru est donc dans un premier temps celui de l'assertion positive A (déjà tranchée et préconstruite) nous sommes en mai. C'est cette assertion qui est reprise par l'énonciateur avec le marqueur que parce qu'elle fait l'objet d'une ré-évaluation énonciative de sa part, en ce qu'elle offre potentiellement matière à débat. La question de la prise en compte du complémentaire linguistique est écartée comme l'indique bien et on est en présence d'une affirmation renforcée : après parcours des deux chemins I (être en mai) et $\mathrm{E}$ (ne pas être en mai) on extrait de l'alternative (être / ne pas être en mai) la valeur positive I. Le parcours a pour issue la confirmation de la situation de la lexis prédiquée dans la zone de validation de départ. On aboutit ainsi à une assertion consensuelle, parfaitement stabilisée car prise en charge par le co-énonciateur comme par l'énonciateur ${ }^{4}$.

11 Cependant le travail de réappropriation énonciative de A par l'énonciateur n'a pas encore atteint son but; on n'est pas dans la même configuration que lorsqu'on a bien tout seul comme en (3) que nous fabriquons pour les besoins de la démonstration :

(3) « Sommes-nous en mai ? » « Oui, nous sommes bien en mai ! "

12 Avec bien que se pose le problème de la mise en relation interlexis : l'énonciateur ne s'intéresse à $\mathrm{A}$ que dans la mesure où $\mathrm{A}$ se voit associée à une autre lexis prédiquée, et c'est à ce sujet qu'il y a conflit intersubjectif; l'on passe de la concession à l'adversativité par le biais d'un nouveau parcours sur $\mathrm{A}$ dans la mesure où $\mathrm{A}$ donne lieu à une classe d'énoncés qualitativement convergents sur un plan fictif, virtuel (par exemple les fleurs ne craignent plus le gel / il fait bon / on éteint le chauffage...) mais au final l'énonciateur choisit de n'en asserter aucun car à ses yeux seul le complémentaire linguistique de l'un d'entre eux convient dans la situation d'énonciation, sur le plan de l'effectif ${ }^{5}$, du certain, à savoir B, il fait froid. On aurait tout aussi bien pu avoir les fleurs craignent encore le gel / on a remis le chauffage en marche... Dans Culioli 1999, on peut lire que : 
«bien (bien que) ou beau (il a beau) sont l'image d'une assertion positive infiniment itérée. On sait, d'ailleurs, que le schéma concessif consiste à tout concéder, ou plutôt à poser que l'on concède tout l'imaginable (il s'agit d'une totalité abstraite, ou faut-il dire idéelle ?) pour mieux affirmer, par contraste adversatif, qu'il reste que (...).» (T. $2: 50)$ préconstruite, qui est finalement asserté en tant qu'issue au parcours effectué sur ce qui apparaît comme une totalité énonciative constituée, hermétique à toute forme d'altérité (être en mai donne lieu à faire bon par exemple). Après le "maintien du parcours » (Culioli 1990, T. 1:121) sur la relation interlexis virtuelle que nous venons d'évoquer, la stabilisation provient de la valeur finalement retenue et assertée par l'énonciateur en B sous la forme d'un "reste " qui réintroduit de l'altérité qualitative dans la situation. Selon nous, ce n'est pas seulement A qui fait l'objet d'une « assertion positive infiniment itérée "; c'est aussi la relation interlexis escomptée à partir de $\mathrm{A}$, telle que H. Wyld (2003 : 30-31) l'a décrite : «q 監 $\rightarrow$ normally (among other class members) pi », où « q » est notre A et «pi » notre NON-B. Quant au « reste », il s'agit de la relation interlexis effectivement assertée par bien que (ou beau) entre A et B, notée « $\mathbf{q}$ 䁌 $\rightarrow$ in fact $\mathbf{p}_{\mathrm{i}}^{\prime}{ }^{\prime} »$ par Wyld 6 .

Ainsi l'opération de parcours n'est pas seulement compatible avec une issue de type « confirmation" / " accord » dans laquelle on aboutit à une opération d'identification au niveau des prises en charge énonciatives, mais elle l'est aussi avec une issue de type " divergence " / " désaccord » où l'opération de différenciation prime contre toute attente sur celle d'identification escomptée! Ainsi, avec bien que on parvient à concilier "parcours " et " accès à l'issue " du fait de l'aboutissement à une relation interlexis stabilisée dans la zone du complémentaire linguistique du domaine notionnel. Considérons à ce sujet les propos d'A. Culioli sur « site et domaine » :

«considérons un domaine de validation et parcourons les occurrences d'une certaine notion (une lexis, pour fixer les idées). Le parcours, à la différence de l'extraction et du fléchage, fait que l'on ne peut (ou veut) distinguer aucune occurrence dans le domaine. Si l'on en distinguait une, elle serait nécessairement située ; si l'on n'en distingue pas une, cela entraîne que les occurrences n'ont pas de site. Or, toute énonciation vise à (re)construire de bonnes formes, c'est-à-dire des occurrences munies d'un site, afin d'obtenir des formes stabilisées. Quatre cas peuvent se présenter :

(a) maintien du parcours, avec recours à autrui (interrogation) ;

(b) maintien du parcours avec relation inter-lexis (concessive, par exemple);

(c) centrage (d'où soit homogénéisation toute personne..., soit haut degré est-il bête ! à côté de qu'est-ce qu'il est bête !).

(d) sortie (respectivement entrée) : interrogation rhétorique.

En (a), c'est la réponse qui stabilisera l'énoncé interrogatif; en (b) le site sera fourni par la clause assertive finale : (...) il reste que ; en (c) la généricité ou l'exclamative éliminent l'absence de stabilité ; en (d), on aboutit à une assertion. » (Culioli 1990, T. $1: 121$; notre surlignage) bien le cas sur le plan de l'effectif, que «nous sommes en mai donne lieu à il fait bon » est bien le cas sur le plan du fictif, il reste que «il fait froid» est le cas sur le plan de l'effectif, et non « il fait bon » comme on pouvait s'y attendre sur le plan du fictif. »

Avec bien que, l'altérité est prise en compte avant d'être définitivement éliminée car il y a repassage par le trajet ordonné de la came qui finit par s'arrêter sur I pour ce qui est de la validation de A par le co-énonciateur comme par l'énonciateur. Cependant, l'itération du 
préconstruit notionnel (être en mai donne lieu à faire bon) ne conduit pas à une issue identique pour les deux instances énonciatives, puisque seule l'issue sur B se concrétise à partir de A sur le plan de l'effectif pour l'énonciateur, tandis que NON-B est associée à la position assignée au co-énonciateur sur le plan du fictif sans donner lieu à une issue effective dans la situation, même si l'énonciateur s'en accommode en tant qu'issue virtuelle dans une autre situation possible.

Culioli signale que tous les énoncés concessifs produisent "cette même opération de "concession" [...], cette relation qui est une relation de "non causalité»: ce qui découle d'un premier terme n'est pas ce à quoi on s'attendait » et en propose la glose suivante: " Je te concède tout ce que tu veux, il reste qu'il fait froid " (2002:172). En complément des énoncés vus en (1), citons ceux de (4):

(4) Nous sommes en mai, il reste qu'il fait froid.

Nous sommes en mai, mais il fait froid.

Nous sommes en mai, pourtant il fait froid.

Quoique nous soyons en mai, il fait froid.

Il fait froid, ça se comprendrait si on n'était pas en mai.

Que l'on soit en mai ou que l'on ne soit pas en mai, qu'est-ce que tu veux que ça me fasse, de toute façon, il fait froid, etc. "

(A. Culioli, $2002: 171$ )

Ces énoncés sont interprétables comme « concessifs » car l'on considère qu'ils s'opposent à une relation d'entraînement attendue entre les notions $\mathrm{A}$, être en mai, et « la négation du terme que l'on associe normalement à A » (Morel 1996: 7), notée NON-B, faire froid, et glosable par « Normalement quand on est en mai, il ne fait pas froid / il fait bon. » :

«Dire [...] que "A est normalement associé à B1" [ = NON-B] suppose une vision préétablie de la relation entre les éléments mis en présence, ou du moins un accord tacite entre les locuteurs sur cette relation. [...] Recourir à la concession, c'est en effet imposer la relation implicite qui lie les énoncés mis en présence. » (Morel, $1996: 7)$

Dans la mesure où, à la lecture (ou à l'énonciation) de ces énoncés, tout énonciateur reconstruit la relation non verbalisée "A entraîne normalement NON-B ", dans la TOE nous parlons alors d'un « préconstruit notionnel» :

«Concession: Relation modale inter-lexis établie par l'énonciateur. La démarche concessive consiste à asserter en même temps comme vraies deux propositions mutuellement exclusives en terme de préconstruction notionnelle, tout en indiquant le fait que l'énonciateur est conscient de la contradiction. [...] Ainsi, la concession apparaît comme l'anti-causalité.

Le terme de concession vient de ce que l'énonciateur qui prend ainsi en charge ce qui peut être considéré comme une contradiction le fait en connaissance de cause, c'est-à-dire en concédant au co-énonciateur l'existence de cette contradiction. » (Groussier et Rivière $1996: 43$ )

Considérons à présent les énoncés anglais de notre corpus.

\section{Etude de though, although et even though}

(2) Although it was April, a freezing wind blew through the streets of the city. (R. Dahl, cité par Deléchelle (1982) et repris par Delmas (1987))

(5) Occasionally, he would drop in on his mother for lunch. These visits, though largely uncommunicative, brought joy to Christine's heart. Fibich, returning from the office, would say, 'Any news today ? Did Toto come?'

(6) And though Christine would too often reply, 'No, nothing today', they both had the warm and comforting feeling that something had been restored. (Anita Brookner, Latecomers, 1988) 
(7) She was a typist in the company run by Hartmann and Fibich, and not a particularly good one, but although they were irritated by her lack of attention and her dubious punctuality, her altogether sunny indifference to the demands of the work they politely asked her to do, they both found themselves hypnotized by her self-importance and waited with genuine interest for her appearance at the office. (id.)

(8) [...] The trail of scent, the spotless cuffs, the white hands, and of course the enhanced awareness of herself that promised an exalted deployment of her attributes, all amused him, beguiled him, and though he privately thought her rather absurd, not altogether serious, and perhaps even a little pathetic, there came a point beyond which he could no longer deny himself the pleasure of her company, or rather, the spectacle of her personality. (id.)

(9) These days the splendid body was more voluminous, the hair dyed to an uncompromising shade of gold. With her new amplitude her bustling demeanour made a more genuine impression. Although still not inclined to take her seriously, Hartmann trusted her absolutely. (id.)

(10) 'I wonder if you've had any trouble with your phone lately. Wires sometimes get crossed. A person tries to call a number, and even though he dials correctly, he gets someone else.'(Paul Auster, The New York Trilogy, 1990)

(11) [...] Somehow, even though everything Hagrid had told him so far was unbelievable, Harry couldn't help trusting him. (J.K. Rowling, Harry Potter and the Philosopher's Stone, 1997)

19 On a vu qu'avec bien que (ou beau) les lexis prédiquées A et B faisait l'objet d'un parcours de leur domaine notionnel respectif, parallèlement à un parcours des positions assignables aux instances énonciatives susceptibles de les prendre en charge.

Il en va de même en anglais, mais l'opération de confirmation (assertion renforcée) dont A est la cible, puis celle dont le préconstruit notionnel (A donne lieu à NON-B) est la cible - sous forme d'une « itération » abstraite de la classe des sujets-asserteurs susceptibles de prendre en charge leur validation avec pour seule issue le fait que tous s'y conforment apparait comme plus ou moins marquée explicitement en anglais :

- non marquée avec though ;

- marquée avec although et even though du fait de leur combinaison avec les marqueurs de parcours all et even.

21 En effet, l'emploi de though est à relier à la particule assertive doch en allemand (si en français). Or contrairement à bien comme en (3) ou à si en français, though n'a pas, en anglais contemporain, d'emploi spécifique pour marquer la confirmation. A travers le morphème TH- de préconstruction though pointe vers un avant contextuel ou partagé. Il s'agit alors simplement de flécher, de reprendre un état de choses préalablement asserté. Le parcours-identification effectué sur la classe des sujets-asserteurs de la validation de A est donc appréhendé à travers son issue ; l'énonciateur nous fait part du résultat de cette opération plutôt que de l'itération elle-même : tous les énonciateurs sans exception reprennent l'assertion de A à leur compte dans la situation; tous les énonciateurs sans exception reconnaissent le bien fondé du préconstruit notionnel (A donne lieu à NON-B) escompté sur le plan du fictif ; en revanche, l'énonciateur asserte B à côté de A sur le plan de l'effectif, se démarquant par là des autres énonciateurs virtuels, représentés par l'image abstraite du co-énonciateur.

On peut gloser though par « et avec ça », « outre cela », comme l'indique G. Bourquin au sujet de l'étymologie du marqueur :

« le mot THOUGH est issu du germanique *Da + ux (cf. got. Dau(h), v.h.a. doch,...),

lui-même déjà constitué de deux segments: Da est une forme d'anaphorique- 
déictique (<ind.eur. ${ }^{*}$ to-) et ux un coordonnant enclitique (<ind.eur. ${ }^{*} \mathrm{k}^{\mathrm{w}}$-, cf. lat. que). Le sens premier de *Daux devait donc être : "et ce(la)", "avec ce(la)" ou "outre ce(la)". On perçoit déjà l'analogie avec le premier segment de ce-pendant et l'itérativité qu'induit tout anaphorique. On pense aussi au français familier : « il fait froid et avec ça il sort !» où «et avec ça » dit en clair ce que dit cryptiquement *Daux (et, partant, THOUGH). » (Bourquin 1984 : 22)

En (5), (6) et (8), on reconstruit, semble-t-il, l'existence du parcours, à partir des lexis prédiquées A parfaitement stabilisées, puis de leurs prolongements qualitatifs vers NON$B$, non susceptibles d'être remis en cause par quelque énonciateur que ce soit. Ce qui peut se gloser par

- pour A : «cela est un fait qu'en (5) these visits were largely uncommunicative, en (6) Christine would too often reply, 'No, nothing today' et en (8) he privately thought her rather absurd, not altogether serious, and perhaps even a little pathetic sur le plan de l'effectif pour tous les énonciateurs. "

- pour le préconstruit notionnel: "cela est un fait qu'en (5) these visits were largely uncommunicative donne lieu à they did not bring joy to Christine's heart, en (6) Christine would too often reply, 'No, nothing today' à they did not feel any better about their relationship with their son et en (8) he privately thought her rather absurd, not altogether serious, and perhaps even a little pathetic à he had no pleasure in her company sur le plan du fictif pour tous les énonciateurs. » En tant que telle l'opération de parcours est implicite.

En revanche, la bifurcation adversative vers $B$, complémentaire linguistique nonpréconstruit, serait frayée en quelque sorte par le même morphème TH- qui ne se contenterait pas seulement de pointer vers l'avant (textuel ou partagé) à la manière d'un anaphorique repéré par rapport à la sphère du co-énonciateur, mais aussi de pointer vers la suite du discours à la manière d'un déictique repéré par rapport à la sphère de l'énonciateur. TH- serait alors relayé par le morphème -OUGH qui lui aussi relie ce qui précède à ce qui va suivre, jusqu'à prendre la valeur explicitement adversative qu'on lui connaît, glosable par « et avec cela » signifiant « outre cela ». L'énonciateur asserte finalement et effectivement B dans la situation d'énonciation : en (5) they brought joy to Christine's heart, en (6) they both had the warm and comforting feeling that something had been restored et en (8) there came a point beyond which he could no longer deny himself the pleasure of her company, or rather, the spectacle of her personality.

Though est donc un terme à la fois associatif et dissociatif, concessif (consensuel) et adversatif, pour lequel l'étape " parcours » est simplement induite sur le trajet parcours des positions assignées aux instances énonciatives $\rightarrow$ identification (par fléchage) $\rightarrow$ différenciation (par bifurcation).

Pour ce qui est de although, A. Culioli précise que :

« «though». A l'origine, c'est le même mot que « doch» en allemand, c'est une particule assertive. Maintenant, ça signifie "quoique ». Donc, c'est une "antiassertion ». Là aussi, ça a basculé. [...] En anglais, à la place de «though", vous pouvez avoir « although ». C'est formé de « all » et de « though ».

Et dans de nombreuses langues, pour indiquer la concession, vous allez avoir " tout ». Et d'ailleurs, en français, on peut dire « tout grand qu'il soit ».

En anglais, donc ce " although », c'est une particule assertive "though », et puis ce « all » devant, qui signifie « tout ». En anglais archaïque, il existe une expression qui est « albeit » ( = all be it) qui veut dire littéralement, «tout qu'il soit ». Vous voyez, on retrouve ça partout. » (Culioli $2002: 172-173$ )

Le quantifieur all est la trace d'une opération de parcours avec totalisation ${ }^{7}$ (Culioli 1995 : 87 ; 1999, T. $3: 178$ ) qui porte ici sur le domaine circonscrit de la validation de A 
comme sur celui du préconstruit notionnel qu'on peut lui associer, domaines susceptibles d'être pris en charge par tout énonciateur : voici matérialisée à travers le marqueur al (l)though la totalité notionnelle et énonciative constituée que nous évoquions précédemment pour bien que, beau et though, à savoir que sur le plan inter-énonciatif toute remise en cause est exclue, et c'est cette totalité qui fait l'objet de l'identification après parcours. L'Intérieur du domaine notionnel est rendu homogène et compact par globalisation $^{8}$ - globalisation engendrée par l'identification effective des points de vue énonciatifs sur l'assertion de A après balayage sur chacun d'eux et le passage vers l'Extérieur n'a donc pas lieu d'être ; engendrée par l'identification fictive des points de vue énonciatifs sur l'assertion du préconstruit notionnel et le passage vers l'Extérieur n'aurait donc pas lieu d'être. On aboutit à la construction explicite de l'exclusion de l'altérité. Selon A. Culioli, «la totalisation implique qu'il n'y a pas de reste; on voit donc que l'on ne saurait restreindre la totalisation à une totalisation énumérative» (1999, T. $3: 178)$. Ce que l'on peut gloser par : en (2), «Tout compte fait / tout bien considéré, pour moi y compris, on ne peut rien retirer au fait que it was April est le cas dans la situation; tout compte fait / tout bien considéré, pour moi y compris, on ne peut rien retirer au fait que it was April donnant lieu à a nice breeze blew through the streets of the city est théoriquement le cas. " De même pour (7) avec they were irritated by her lack of attention and her dubious punctuality, her altogether sunny indifference to the demands of the work they politely asked her to do - entraînant normalement they both could not wait to get rid of her - et pour (9) avec Hartmann was still not inclined to take her seriously - entraînant normalement he did not quite trust her.

Ce renchérissement du consensus autour de $\mathrm{A}^{9}$ et de son préconstruit notionnel n'empêche pas la bifurcation adversative d'avoir lieu en B malgré tout : sont effectivement le cas en (2) a freezing wind blew through the streets of the city, en (7) they both found themselves hypnotized by her self-importance and waited with genuine interest for her appearance at the office et en (9) Hartmann trusted her absolutely.

Even est également un marqueur de non-discontinuité ${ }^{10}$ et indique un parcours avec homogénéisation dans la mesure où se trouve incluse in extremis à l'Intérieur du domaine notionnel une occurrence préconstruite comme devant appartenir à l'Extérieur : l'Intérieur est donc homogénéisé jusqu'au dernier point (Culioli 1995 : 85-87 ; 1999, T. 3 : 177-178). Ainsi on part d'une altérité de fondation, et donc préconstruite, pour aboutir à son aplanissement (Gauthier 1997: 122-124), selon la glose suivante pour ce qui est de even though : en (10), "Quand bien même on penserait le contraire, pour moi y compris, le fait que he dials correctly est effectivement le cas; quand bien même on penserait le contraire, pour moi y compris, le fait que he dials correctly donne lieu à he gets the right person est théoriquement le cas " et en (11) everything Hagrid had told him so far was unbelievable - entraînant normalement Harry couldn't trust him at all. On construit ici encore explicitement l'exclusion de l'altérité, et par là une assertion homogène et donc renforcée, du fait de l'inclusion de l'énonciateur lui-même dans la classe des sujetsasserteurs, et c'est cet aplanissement des positions assertives des énonciateurs qui est mis en évidence après parcours. A propos d'un autre marqueur comportant " quand » et «même », A. Culioli précise que « le concessif quand même » est « la trace de l'opération de parcours »: "on parcourt mentalement les obstacles divers (biais négatif)» $(1990$, T. $1: 147)$. 

de l'analyse, on peut supposer que all et even ne portent pas seulement sur la confirmation inter-énonciative de A, mais également sur le renforcement de la force argumentative du préconstruit notionnel associé à la lexis prédiquée A qui tend à éliminer l'issue du parcours des valeurs I et $\mathrm{E}$ du domaine de validation de la lexis prédiquée B sur E (à savoir B) au profit de l'issue sur I (à savoir NON-B) escomptée. Or, il n'en est rien puisque all et even contribuent également à renforcer la bifurcation vers la validation de B assertée par l'énonciateur dans la situation, glosable par «il reste malgré tout que B est le cas » et «il reste quand même que B est le cas». Ce qui conduit à une confortation de la partition opérée sur la classe des instances énonciatives au sujet de l'assertion de B en Sit.

31 Cela permet de rendre compte des remarques souvent rencontrées dans les grammaires qualifiant although et even though d' "intensifs" de though, ce qui s'explique selon G. Bourquin (1984: 33) par le fait que "l'itératif se mue en intensif.» Cela montre que la façon de construire une totalité notionnelle et énonciative autour de A peut être plus ou moins marquée au niveau des différentes composantes du marqueur concessif lui-même, sans que cela empêche le fait que dans la concessive, il y ait toujours un reste. Ce que A. Culioli évoque en termes de «relation d'inefficacité » $(1995: 88 ; 2002: 173)$ :

"pour concéder, c'est-à-dire pour dire que quelque chose va à l'encontre de ce qu'on attendait, il faut: poser d'un côté un premier état de choses, que l'on confirme, dont on dit «c'est le cas »; en tirer que l'on s'attendrait normalement à ce que quelque chose en découle; et tout ça pour mieux dire : ce qui en découle, ce n'est pas ce à quoi on s'attendait. Donc, il faut tout concéder, pour mieux dire que c'est en vain, que ce n'est pas efficace, comme dans :

Tu as beau crier tant que tu veux, tu t'attends à ce que je cède, eh bien je ne céderai pas.

Ça, c'est une forme de concessive : « crie tant que tu veux », qui signifie bien «quel que soit le volume de tes cris, bien que tu cries, je ne céderai pas». (Culioli 2002: 173)

\section{En guise de conclusion}

Le type de parcours dont il est question dans les énoncés concessifs est donc induit par la remise en cause énonciative d'une RP déjà assertée et se matérialise par le balayage ordonné/orienté des deux zones du domaine notionnel, l'Intérieur puis l'Extérieur, à partir d'une position hors-domaine, en vue de valider telle RP, aboutissant par là à sa situation dans une zone à l'exclusion de l'autre. Au niveau de $\mathrm{A}$, on construit une assertion confirmée, tandis qu'au niveau de B on construit une bifurcation vers une valeur autreque-préconstruite car autre-que-escomptée. En effet, la configuration concessive antéposée se prête particulièrement bien à la mise au jour d'une opération d'altérité 
qualitative dans la mesure où l'on assiste à la réintroduction d'une instabilité qualitative là où était préconstruite une totalité notionnelle et énonciative stable, de nature «abstraite » ou « idéelle » (Culioli 1999, T. 2 : 50 et aussi Culioli 2002 : 171-173), de sorte que dans la concessive, il y a toujours un reste qui constitue l'issue au parcours.

L'opération de parcours s'applique tout autant aux positions assignées aux instances énonciatives et l'issue sur une valeur I consensuelle en A se voit combinée à une issue sur une valeur adversative (E) en $B$, à travers laquelle s'exprime le point de vue de l'énonciateur de manière prépondérante dans l'échange inter-énonciatif. On aboutit ainsi à la caractérisation de la relation concessive-adversative en termes de construction d'une relation de concomitance sans la consécution (Filippi-Deswelle 2003) basée sur une opération d'identification-différentiation après parcours, engendrée par la mise en relation interlexis. Avec bien que, though, although et even though, la dimension adversative prime. Notons qu'avec though, le parcours est davantage induit - ce que l'on peut représenter par un marqueur zéro comme suit : $\varnothing$ though - tandis qu'il est explicitement construit par all et even dans although et even though ou par bien dans bien que :

- $\emptyset$ though

- although

- even though

- bien que

L'opération de parcours n'est ici qu'une étape sur le trajet vers une issue effective stabilisée dans la situation d'énonciation - issue introductrice d'altérité contre toute attente.

\section{BIBLIOGRAPHIE}

Adamczewski, H. et C. Delmas. 1982. Grammaire linguistique de l'anglais, Paris : Armand Colin collection U

Bonnard, H. 1981. Code du français courant, Paris : Magnard.

Bourquin, G. 1984 « Discours de langue et discours de parole : les énoncés concessifs », RANAM

XVII, p. 7-33.

Bouscaren, J. et J. Chuquet. 1987. Grammaire et textes anglais Guide pour l'analyse linguistique, Paris : Ophrys.

Culioli, A. 1975/1976 Recherche en linguistique. Théorie des Opérations Enonciatives, Transcription du séminaire de D.E.A. par les étudiants, Université Paris VII.

Culioli, A. 1990. Pour une linguistique de l'énonciation, Opérations et représentations, Tome 1, collection l'Homme dans la langue animée par Janine Bouscaren, Gap : Ophrys.

Culioli, A, 1999. Pour une linguistique de l'énonciation, Formalisation et opérations de repérage, Tome 2, collection l'Homme dans la langue animée par Janine Bouscaren, Gap, Paris : Ophrys. 
Culioli, A, 1999. Pour une linguistique de l'énonciation, Domaine notionnel, Tome 3, collection l'Homme dans la langue animée par Janine Bouscaren, Gap, Paris : Ophrys.

Culioli, A. 1999. "Even though, even if ; as though, as if ", Linguistique et Didactique, Cahiers Charles Vn • 19, Université Paris 7 - Denis Diderot (1995), p. 85-91. Aussi dans Pour une linguistique de l'énonciation : domaine notionnel Tome 3, Paris : Ophrys, p. 177-181.

Culioli, A 2002. Variations sur la linguistique, Klincksieck.

Danon-Boileau, L. 1995. « Symbolisation, fonction du langage et statut du sujet entre psychanalyse et linguistique ", Langues et langage, Problèmes et raisonnement en linguistique, Mélanges offerts à Antoine Culioli, PUF, p. 553-564.

Delmas, C. 1987. Structuration abstraite et chaîne linéaire en anglais contemporain, Thèse, Paris, «Collection Linguistique » publiée par la Société de linguistique de Paris LXXV, p. 259-262.

Ducrot, O. 1982. « La notion de sujet parlant », Recherche sur la philosophie et le langage, $n^{\circ} 2$, Université de Grenoble, p. 65-93.

Filippi, C. 1997. «Though et la relation de concession : opération énonciative et jeu sur le domaine notionnel », La notion, C. Rivière et M.-L. Groussier (éd.), Paris : Ophrys, p. 91-98.

Filippi, C. 1998. « La relation de concession : étude contrastive de though en anglais et de bien que, même si et encore que en français ", Linguistique contrastive et traduction Tome 4, Paris : Ophrys, p. 25-60.

Filippi-Deswelle, C. 1999. " Etude énonciative de if et though antéposés ", L’hypothétique, LINX 41, A. Trévise (éd.), Nanterre : Université Paris X-Nanterre, p. 75-91.

Filippi-Deswelle, C. 2000. La relation dite de " concession " :étude de THOUGH, ALTHOUGH, EVEN THOUGH et EVEN IF antéposés en anglais contemporain, Villeneuve d'Ascq : Presses Universitaires du Septentrion.

Filippi-Deswelle, C. 2003. «Though antéposé : de la concession à l'adversativité", La subordination en anglais, Une approche énonciative, A. Celle et S. Gresset (éd.), Collection Interlangues, Linguistique et Didactique, Toulouse : Presses Universitaires du Mirail, p. 217-246.

Filippi-Deswelle, C. Colloque « Langues dominantes/Langues dominées » de Novembre 2003, Université de Rouen (ERAC), à paraître aux Presses Universitaires de Rouen et du Havre.

Filippi-Deswelle, C. « Le « domaine notionnel » d'Antoine Culioli (ou comment la géographie (méta)linguistique à l'œuvre dans nos représentations topologiques abstraites régule notre activité langagière) » Colloque « Géographies imaginaires » de Mars 2005, Université de Rouen (ERAC/ACIE), à paraître aux Presses Universitaires de Rouen et du Havre.

Gauthier, A. 1981. Opérations énonciatives et apprentissage d'une langue étrangère en milieu scolaire, l'anglais à des francophones, Les langues modernes.

Gauthier, A. 1997. «EVEN, JUST et le domaine notionnel », La notion, Paris : Ophrys, p. 121-128.

Guillemin-Flescher, J. 2003. « Sujet énonciateur, sujet de l'énoncé dans la relation d'interlocution fictive », Le sujet, J.-M. Merle (éd.), Bibliothèque des Faits de Langue, Paris : Ophrys, p. 183-192.

Morel, M.-A. 1996. La Concession en français, Paris : Ophrys.

Huart, R. 1997. «All : question de portée », Cahiers de Recherche T.7, J. Bouscaren (dir.), Paris : Ophrys.

Oxford English Dictionary, vol. XI., T. U., though p. 339-340. 
Ranger, G. 1998. Les Constructions concessives en anglais : une approche énonciative, Cahiers de recherche - numéro spécial, Paris : Ophrys.

Robert, S. 1994. « Sur le rôle du sujet énonciateur dans la construction du sens : liens entre temps, aspect et modalité ", Subjecthood and Subjectivity, The status of the subject in linguistic theory, M. Yaguello (éd.), Paris : Ophrys , p. 209-229.

Simonin, J. 1984. « De la nécessité de distinguer énonciateur et locuteur dans une théorie énonciative ", Revue du DRLAV 30, Paris VIII, p. 55-62.

Trévise, A. 1999. «A propos des repérages fictifs : variété des formes et construction du sens ", L’hypothétique, LINX 41, A. Trévise (éd.), Nanterre : Université Paris X-Nanterre, p. 39-59.

Trévise, A. 2003. «A propos de quelques relations inter-énoncés : hypotaxe, parataxe, asyndète et construction du sens ", La subordination en anglais, Une approche énonciative, A. Celle et S. Gresset (éd.), Collection Interlangues, Linguistique et Didactique, Toulouse : Presses Universitaires du Mirail, p. 51-68.

de Vogüé, S. 1985. Référence, prédication, homonymie : le concept de validation et ses conséquences sur une théorie des conjonctions, Thèse de doctorat, Université Paris 7.

de Vogüé, S. 1986. « La conjonction si et la question de l'homonymie », BULAG 13, Université de Besançon, p. 109-189.

de Vogüé, S. 1990. «Inférence sous inférence », Le gré des langues n 1, L’Harmattan, p. 7-24.

de Vogüé, S. 1999. « Le champ des subordonnées dites conditionnelles du français : conditions, éventualités, suppositions et hypothèses », L'hypothétique, LINX 41, A. Trévise (éd.), Nanterre : Université Paris X-Nanterre, p. 93-118.

Wyld, H. 2001. Subordination et énonciation, Cahiers de Recherche en Grammaire Anglaise, Numéro spécial, Paris : Ophrys.

Wyld, H. 2003. « Adverbial clauses : an enunciative approach », La subordination en anglais, Une approche énonciative, A. Celle et S. Gresset (éd.), Collection Interlangues, Linguistique et Didactique, Toulouse : Presses Universitaires du Mirail, p. 15-38.

\section{NOTES}

1. Pour en savoir plus sur le « domaine notionnel », cf. Culioli 1990 T. 1, 1999 T. 2 et T. 3 ainsi que Filippi-Deswelle mars 2005 (à paraître).

2. Cf. Guillemin-Flescher (2003: 183, note 1) : "L'énonciateur est [...] envisagé comme point de référence abstrait dans le calcul du temps, de l'espace et des personnes et comme support des modalités." et Culioli $(1990: 110)$ : "un autrui fictif, c'est-à-dire un co-énonciateur qui n'est pas un interlocuteur." De Vogüé (1986 : 159-160) parle dans ce cas d'"énonciateur fictif" au sujet de l'asserteur de A. Voir aussi Bouscaren et Chuquet (1987 : 133) sur le repérage fictif, noté * (étoile) et sur la distinction entre énonciateur-origine et énonciateur-asserteur (1987: 144-145). Les termes changent d'un auteur à l'autre: Ducrot (1982: 74-76) parle de "locuteur" pour le "responsable de l'énoncé" et d'"énonciateur(s)" pour le(s) "responsables de tel ou tel acte de langage accompli dans l'énonciation", tandis que Simonin (1984: 56) parle respectivement de l'énonciateur" comme "paramètre intervenant dans la construction des valeurs référentielles" et de "locuteur" comme "support des opérations de modalisation".

3. Pour un plus long développement à ce sujet, cf. Filippi-Deswelle novembre 2003 (à paraître). 
4. Sur la discussion autour de repère stable ou de repère constitutif pour A, cf. Ranger 1998 et Filippi-Deswelle 2003.

5. Cf. Trévise 1999 et 2003.

6. Selon G. Ranger, l'énoncé concessif opère "la déconstruction du lien inférentiel" (1998: 30). H. Wyld parle dans ce cas de "deviative actualisation (Fr. "brisage")" (2003 : 30). Cf. Wyld (2001 : 178-193), plus particulièrement les pages 180-182 consacrées au «double mode d'existence de la relation causale préconstruite : existence virtuelle vs existence actualisée " où "l'entérinage » s'oppose alors au « brisage » dans la concession.

7. Sur la totalisation par itération pour all et tout, G. Bourquin précise qu'il s'agit d'un «parcours en intension" glosable par «itérez aussi souvent que vous pouvez jusqu'à épuisement des possibles [...] ; faisons retour indéfiniment sur ce thème. » $(1984: 12 ; 16)$. Sur le rôle de all en tant que marqueur intensif dans la concession, R. Huart indique que all «assume alors une valeur modale d' "intensification », car le parcours marqué par all équivaut à une identification, avec l'effet d'une assertion forte «certes », « il est parfaitement vrai que », soit : « je ne nie pas que » (de nouveau l'exclusion de l'altérité)» (1997: 88-89).

8. Pour G. Deléchelle, « la valeur concessive est due au contraste entre l'itération/globalisation que marque all et le contenu de $q$, ce qui a pour effet de renforcer de dernier » (1982:127), où « $q$ » équivaut à notre lexis $\mathrm{A}$.

9. Pour G. Ranger, all est la trace d'une « opération d'indifférenciation par laquelle on établit une continuité sur une classe d'occurrences » et lors son association avec though « l'indifférenciation porte sur les instances énonciatives de la prise en charge de la lexis » (1998 : 71-72).

10. Selon G. Ranger, even « est fondamentalement un marqueur d'identification que l'on pourrait décrire négativement comme marquant l'élimination d'une discontinuité [...] potentielle » (1998: 81-82; 85).

11. A savoir $x$, à l'exclusion de autre-que- $x$ dans Filippi-Deswelle 1998 [2000]. Or il n'en va pas de même avec even if, en raison de la combinaison de l'adverbe intensif even et de la conjonction de subordination if qui construit la conjonction complexe à valeur concessive even if. Ces deux marqueurs indiquent, chacun à leur façon, la prise en compte, et donc la réintroduction, de l'altérité dans le domaine de validation de $\mathrm{A}$ : avec even if, on travaille sur I et $\mathrm{E}$, à savoir sur l'ensemble du construit notionnel représenté par la relation prédicative évoquée en $\mathrm{A}$, noté $\mathrm{A}$ ( $a$, autre-que-a). Pour une analyse distributionnelle détaillée mettant en cause la totale interchangeabilité de ces marqueurs concessifs, cf. Ranger 1998 ainsi que Filippi-Deswelle 1998 [2000] et novembre 2003 (à paraître, qui traite en particulier des limites de l'interchangeabilité de though et de even if).

\section{RÉSUMÉS}

Je souhaiterais participer à la discussion autour de l'opération de parcours dans son application dans le cadre de la relation inter-lexis. Plus précisément je voudrais aborder sa mise en œuvre au sein des énoncés concessifs en though, although et even though sous la forme spécifique d'une opération d'itération portant sur un domaine qu'il conviendra d'identifier avec précision et qui trouve son issue dans l'assertion d'une relation contraire à l'attente. L'opération concessive permet la réintroduction d'une instabilité qualitative là où était préconstruite une totalité constituée (de nature abstraite ou «idéelle » selon Culioli 1999 T. 2 et aussi 2002), de sorte que 
dans la concessive, il y a toujours un reste. Outre la mise au jour de cette opération d'altérité qualitative (Filippi-Deswelle 2003) commune aux marqueurs concessifs, il sera intéressant d'étudier l'opération de parcours propre aux marqueurs all et even (associés à though dans l'interprétation concessive), parcours totalisateur pour all et parcours homogénéisateur pour even, afin de préciser leur rôle dans la constitution d'un paradigme concessif restreint. J'essaierai de montrer brièvement comment, selon la formulation de Bourquin (1984), «l'itératif se mue en intensif. »

I wish to discuss the concept of scanning in connection with concessive utterances with "though", "although" and "even though" in which scanning appears as a specific form of an operation of iteration on a domain that should be carefully identified - an operation finding its resolution in the assertion of a predicative relationship that contradicts what could be expected in the situation of utterance. The concessive operation makes it possible to re-introduce a qualitative unstability where there is initially a preconstructed (abstract, ideational) wholeness, in such a way as there is always a remaining trace. In addition to the disclosure of this operation of qualitative otherness (Filippi-Deswelle 2003), which is a factor concessive markers have in common, it will be interesting to study the scanning operation that is specifically associated with the markers "all" and "even" linked with "though" in the concessive interpretation (totalizing scanning for "all" and homogenizing scanning for "even") in order to clarify their roles in the building up of a restricted concessive paradigm. I will endevaour to show briefly how "the iterative turns into the intensive" (Bourquin, 1984).

\section{INDEX}

Mots-clés : concession, parcours, issue, (co-)énonciation, altérité, intensif

\section{AUTEUR}

\section{CATHERINE FILIPPI-DESWELLE}

Université de Rouen 